\title{
The Association Between Inflammation and Angiogenesis in Human Pancreatic Adenocarcinoma
}

\author{
Cristiana Tanase ${ }^{1,2}$, Laura Georgiana Necula ${ }^{1,2,3}$, Ana Iulia Neagu ${ }^{1,3}$, Radu Albulescu ${ }^{1,2,4}$
}

${ }^{1}$ Titu Maiorescu University, Faculty of Medicine, Bucharest, Romania

${ }^{2}$ Victor Babes National Institute of Pathology, Bucharest, Romania

${ }^{3}$ Stefan S. Nicolau Institute of Virology, Bucharest, Romania

${ }^{4}$ National Institute for Chemical Pharmaceutical Research \& Development, Bucharest,

Romania

\section{ABSTRACT}

Pancreatic cancer represents one of the deadliest cancers with a high mortality rate, characterized by an aggressive local invasion and early metastases. This malignancy is poorly responsive to chemotherapy or chemo-radiotherapy. Several proangiogenic factors were associated with pancreatic carcinogenesis, highlighting the importance of using antiangiogenic agents as therapeutic strategy. Further studies suggest the involvement of chronic inflammation in angiogenesis processes in pancreatic carcinogenesis and inflammatory cytokines should be considered as potential biomarkers, as well as therapeutic targets in pancreatic cancer patients.

Key words: pancreatic cancer, angiogenesis, cytokines, therapy

\section{INTRODUCTION}

Pancreatic cancer represents one of the most aggressive and lethal malignancies, having an incidence closely equal to the mortality rate. This poor prognosis is mainly owed to the advanced stage of disease at the time of diagnosis. Most patients with pancreatic cancer present local invasion or micrometastatic tumor cells at presentation (1-3). 337,872 new pancreatic cancer cases and 330,391 deaths were estimated in 2012 worldwide (4). Gemcitabine, the first-line chemotherapeutic drug for locally advanced or metastatic pancreatic cancer, improves the quality of life and prolongs survival by 5-6 months (5).

Recently completed clinical trials investigated new possible chemotherapeutic alternatives which might improve the overall survival. Some of these drugs - used alone or in combination are already approved agents for metastatic pancreatic cancer treatment: Erlotinib (a reversible tyrosine kinase inhibitor), MM-398 (nanoliposome-encapsulated irinotecan), Abraxane (protein-bound paclitaxel-amitotic inhibitor) used in combination with Gemcitabine and FLOFIRINOX (a combination of 4 drugs-oxaliplatin,

Corresponding author: Cristiana Tanase, MD, PhD Professor, Titu Maiorescu University, E-mail: bioch@vbabes.ro
Received: 25.04.2016 Accepted: 07.06.2016

Copyright (c) Celsius Publishing House 
irinotecan, fluorouracil, and leucovorin) (6-8).

Most pancreatic tumors are located in the exocrine pancreas, with pancreatic ductal adenocarcinoma (PDAC) representing approximately $95 \%$ of pancreatic cancers. The main risk factors for pancreatic cancer are smoking, type 2 diabetes mellitus, obesity and pancreatitis, and family history (9). Chronic inflammatory conditions mediated by pro-inflammatory cytokines and their receptors, reactive oxygen species, up-regulated proinflammatory pathways, and immune cell infiltrates are associated with pancreatic carcinogenesis (1).

Even though many studies were focused on molecular characterization of PDAC trying to identify specific therapeutic targets, the best therapeutic options for these patients are surgery and cytotoxic therapies. Nevertheless, sustained by several factors, such as tumor microenvironment, hypoxia, post-transcriptional gene regulation and somatic mutations, PDAC cells exhibit chemo-resistance (10).

\section{ANGIOGENESIS AND INFLAMMATORY FACTORS}

One of the most significant therapeutic approaches is the use of antiangiogenic agents, but even if the majority of PDAC express high levels of proangiogenic factors, the tumor vasculature is abnormal and poorly perfused with blood, restricting the chemotherapeutic penetration (11). Compared to other solid tumors, pancreatic cancers are poorly vascularized and this particularity might explain their aggressiveness and resistance to chemotherapy (12).

However, the neoangiogenesis process is amplified in pancreatic cancers. Intratumoral microvessel density is increased and seems to be an independent prognostic factor for survival in pancreatic cancer patients. Several proangiogenic factors were associated with pancreatic carcinogenesis, including EGFR (epidermal growth factor receptor), VEGF (vascular endothelial growth factor), bFGF (basic fibroblast growth factor) and IL-8 (13).

Angiogenesis represents a critical process for tumor development, being regulated by several different growth factors. VEGF (VEGF-A), VEGF-B, VEGF-C and VEGF-D, VEGF-F, PIGF (placental growth factor), and their receptors VEGFR-1, VEGFR-2 and VEGFR-3 are the most important angiogenic growth factors, involved in angioblast differentiation and tube formation. Expression level of VEGF represents an important marker for the detection of angiogenic diseases. VEGF aberrant expression was identified in most types of digestive cancers, including pancreatic cancer, being considered a diagnostic marker and a poor prognostic factor of the disease (14). In vitro studies demonstrated that VEGF stimulation led to a metabolic transition from mitochondrial oxidative phosphorylation to glycolysis in pancreatic cancer cell lines via HIF1 $\alpha$ up-regulation (15).

EGFR signaling pathway plays a critical role in pancreatic cancer development; several combinations of EGFR inhibitors with other agents resulted in the inhibition of angiogenesis and cell growth. For example, combination of erlotinib with gemcitabine presented statistically significance in overall-survival (16). Moreover, inhibition of VEGF/EGFR pathway seems to reverse radio-resistance (17).

A recent study of Khan et al. revealed an indirect effect of gemcitabine treatment on angiogenesis; it demonstrated that Gemcitabine induces expression of angiogenesis-associated cytokines in pancreatic tumor cells, among which IL-8 proved the highest levels of induction. The up-regulation mechanism of IL- 8 transcription in pancreatic cancer cells involves activation of NF-KB and HIF- $1 \alpha$; IL- 8 determines endothelial cell proliferation, survival, migration and angiogenesis (18).

Previous studies evaluated circulating concentration of two proangiogenic factors VEGF and bFGF in pancreatic cancer patients and healthy controls (19-22). Increased serum levels of VEGF and bFGF in cancer patients significantly correlate with tumor diameter, stage of disease, standard proliferative marker, Ki67, and conventional markers CA $19-9$ and CEA. A possible explanation for the simultaneous increase of these two molecules could be their closely related downstream signaling pathways. EGFR pathway activation increases the production of angiogenic proteins in tumor cells VEGF and bFGF $(23,24)$. Although they are not specific to pancreatic cancer, soluble VEFG and bFGF might be included in the pancreatic cancer biomarker panel as a valuable tool for detection of early recurrence and monitoring of therapy. In an attempt to find a relevant serum marker/set of markers for pancreatic cancer, several other angiogenic molecule and growth factors have been proposed. Among them EGFR (25), IL-8 (26), the ratio of VEGF /VEGFR-1 (27), TGF- $\beta$ (28) and MIC-1 $(29,30)$ showed a significant association with pancreatic cancer. Extensive research have been performed regarding the local/tissue biomarkers: angiogenic factors [e.g. EGF, VEGF, heparanase, cathepsins $(31,32)]$, matrix metalloproteinase 7, fibroblast activation protein $(33)$, caveolin-1 $(34,35)$ are only a few proteins whose increased expression in pancreatic cancer tissue correlates with an unfavorable prognostic.

Recent studies reported increased circulating levels of proangiogenic factors VEGF and bFGF as potential prognostic biomarkers in PDAC patients compared with 
healthy individuals. bFGF, VEGF, PDGF-A, and Ang-1 levels were also associated with lymph node metastasis in PDAC $(22,36)$.

Nevertheless, clinical trials on bevacizumab, a monoclonal antibody against VEGF and Cetuximab, a monoclonal antibody against EGFR, failed to establish a survival advantage of anti-angiogenic therapy for patients with pancreatic cancer. Inflammatory factor overexpression seems to represent one of the mechanisms by which pancreatic tumors progress and become refractory to cytotoxic and antiangiogenic therapies (fig. 1)(36). Recent studies reported a significant correlation between pro-inflammatory cytokines IL-6, TNF- $\alpha$, IL-1 $1 \beta$, IL-10 and serum concentration of VEGF and bFGF. IL-6, IL-1 $\beta$, and TNF- $\alpha$ levels were also significantly correlated with circulating levels of CA 19-9 while IL-8, IL-10, TNF- $\alpha$ levels were significantly correlated with CEA levels in the patients with PDAC. Tumor volume was significantly correlated with IL-1 $1 \beta$, IL-10 and VEGF levels; all the inflammatory markers analyzed, as well as VEGF and bFGF levels, were correlated with tumor stage (1). Another biomarker with potential prognostic value in PDAC patients seems to be circulating IL-8. IL- 8 levels were significant associated with worse survival, being linked with carcinogenesis by promotion of angiogenesis and metastasis, via mitogen-activated protein kinase pathway; IL-8 could be considered a potential target for combination therapy in PDAC (37-39). TNF- $\alpha$ level was found to be increased in the sera of patients with PDAC compared with those of healthy individuals. Moreover, elevated serum levels of TNF- $\alpha$ were correlated with tumor grade and overall survival in patients with PDAC, probably facilitating recurrent tumor growth (40). TNF- $\alpha$ is also involved in metastasis process, serum TNF- $\alpha$ being elevated in patients with metastatic PDAC compared with non-metastatic disease (41). Interestingly, anti-TNF- $\alpha$ treatment (Infliximab) resulted in a decreased serum VEGF concentration in patients with rheumatoid arthritis (42). In PDAC patients a positive correlation between VEGF and bFGF serum levels was identified; these results suggest that future therapies can target TNF- $\alpha$ in combination with other treatments in order to delay tumor progression in PDAC (1). Serum IL-1 $\beta$ was also associated with circulating VEGF and bFGF levels in PDAC patients, acting as promoter of tumor angiogenesis, invasiveness and metastasis (43).

Many studies reported the potential role of inflammatory cytokines in tumor resistance to therapy suggesting that blocking cytokines may sustain the efficacy of anticancer agents (44). Therefore, the levels of many inflammatory cytokines are significantly increased

\section{TUMOR MICROENVIRONMENT - INFLAMMATORY CELLS AND MACROPHAGES / STROMAL CELLS}
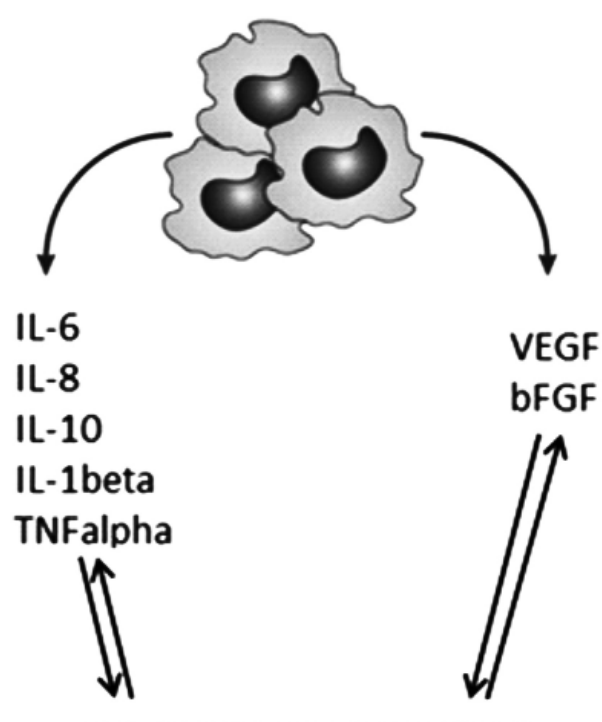

TUMOR ANGIOGENESIS

Figure 1 - Interrelationship between tumor microenvironment and tumor angiogenesis

in the circulation of patients with PDAC. Moreover, the concentrations of inflammatory cytokines are associated with proangiogenic factor levels and both are correlated with poor outcome in PDAC patients (45). An increasing number of studies sustain that inflammatory cytokines can directly promote angiogenesis, as well as induce expression of proangiogenic factors in $\operatorname{PDAC}(39,46)$.

\section{FUTURE PERSPECTIVES}

Angiogenesis has a significant contribution in pancreatic cancer aggressiveness; evaluation of inflammatory cytokine levels combined with angiogenic biomarkers may improve early diagnosis performance, may predict recurrence and survival rate and also tailor the management of personalized therapy in pancreatic cancer patients.

\section{Author's contribution}

All authors have contributed equally to this paper.

\section{Acknowledgements}

This study was financially supported by EEA - JRP Romania - Norvegia no. 4SEE/30.06.2014. 


\section{REFERENCES}

1. Dima S0, Tanase C, Albulescu R, Herlea V, Chivu-Economescu M, Purnichescu-Purtan R, et al. An exploratory study of inflammatory cytokines as prognostic biomarkers in patients with ductal pancreatic adenocarcinoma. Pancreas. 2012 Oct;41(7):1001-7.

2. Qiu M, Qiu H, Jin Y, Wei X, Zhou Y, Wang Z, et al. Pathologic Diagnosis of Pancreatic Adenocarcinoma in the United States: Its Status and Prognostic Value. J Cancer. 2016 Mar;7(6):694-701.

3. Bimonte S, Leongito M, Granata V, Barbieri A, Del Vecchio V, Falco M, et al. Electrochemotherapy in pancreatic adenocarcinoma treatment: pre-clinical and clinical studies. Radiol Oncol. 2016 Feb;50(1):14-20.

4. Torre LA, Bray F, Siegel RL, Ferlay J, Lortet-Tieulent J, Jemal A. Globa cancer statistics, 2012. CA Cancer J Clin. 2015 Mar;65(2):87-108

5. Assifi MM, Hines 0J. Anti-angiogenic agents in pancreatic cancer: a review. Anticancer Agents Med Chem. 2011 Jun;11(5):464-9.

6. Conroy T, Desseigne F, Ychou M, Bouche 0, Guimbaud R, Becouarn Y, et al. FOLFIRINOX versus gemcitabine for metastatic pancreatic cancer. N Engl J Med. 2011 May;364(19):1817-25.

7. Thota R, Pauff JM, Berlin JD. Treatment of metastatic pancreatic adenocarcinoma: a review. Oncology (Williston Park). 2014 Jan;28(1): 70-4

8. Ur Rehman SS, Lim K, Wang-Gillam A. Nanoliposomal irinotecan plus fluorouracil and folinic acid: a new treatment option in metastatic pancreatic cancer. Expert Rev Anticancer Ther. 2016 May;16(5):48592.

9. Amundadottir LT. Pancreatic Cancer Genetics. Int J Biol Sci. 2016 Jan 12(3):314-25

10. Chand S, O'Hayer K, Blanco FF, Winter JM, Brody JR. The Landscape of Pancreatic Cancer Therapeutic Resistance Mechanisms. Int J Biol Sci. 2016 Jan;12(3):273-82

11. Jain RK, Duda DG, Willett CG, Sahani DV, Zhu AX, Loeffler JS, et al. Biomarkers of response and resistance to antiangiogenic therapy. Nat Rev Clin Oncol. 2009 Jun;6(6):327-38.

12. Neesse A, Michl P, Frese KK, Feig C, Cook N, Jacobetz MA, et at. Stromal biology and therapy in pancreatic cancer. Gut. 2011 Jun; 60(6): 861-8.

13. Tanase CP, Neagu M, Albulescu R, Hinescu ME. Advances in pancreatic cancer detection. Adv Clin Chem. 2010;51:145-80.

14. Costache MI, Ioana M, lordache S, Ene D, Costache CA, Saftoiu A. VEGF Expression in Pancreatic Cancer and Other Malignancies: A Review of the Literature. Rom J Intern Med. 2015 Sep;53(3):199-208.

15. Shi S, Xu J, Zhang B, Ji S, Xu W, Liu J, et al. VEGF Promotes Glycolysis in Pancreatic Cancer via HIF1alpha Up-Regulation. Curr Mol Med. 2016;16(4):394-403

16. Nedaeinia R, Avan A, Manian M, Salehi R, Ghayour-Mobarhan M. EGFR as a potential target for the treatment of pancreatic cancer: dilemma and controversies. Curr Drug Targets. 2014;15(14):1293301.

17. Silvestris N, Gnoni A, Brunetti AE, Vincenti L, Santini D, Tonini G, et al Target therapies in pancreatic carcinoma. Curr Med Chem. 2014 21(8):948-65

18. Khan MA, Srivastava SK, Bhardwaj A, Singh S, Arora S, Zubair $H$, et al. Gemcitabine triggers angiogenesis-promoting molecular signals in pancreatic cancer cells: Therapeutic implications. Oncotarget. 2015 Nov;6(36):39140-50.

19. Karayiannakis AJ, Bolanaki H, Syrigos KN, Asimakopoulos B Polychronidis A, Anagnostoulis S, et al. Serum vascular endothelia growth factor levels in pancreatic cancer patients correlate with advanced and metastatic disease and poor prognosis. Cancer Lett. 2003 May 8;194(1):119-24

20. Misek DE, Patwa TH, Lubman DM, Simeone DM. Early detection and biomarkers in pancreatic cancer. J Natl Compr Canc Netw. 2007 Nov; 5(10):1034-41.

21. Kobayashi A, Yamaguchi T, Ishihara T, Ohshima T, Baba T, Shirai Y, et al. Usefulness of plasma vascular endothelial growth factor in the diagnosis of pancreatic carcinoma: differential diagnosis, tumor progression, and patient survival. Pancreas. 2005 Jul;31(1):74-8.

22. Pistol-Tanase C, Raducan E, Dima SO, Albulescu L, Alina I, Marius P, et al. Assessment of soluble angiogenic markers in pancreatic cancer. Biomark Med. 2008 0ct;2(5):447-55.

23. Baker $\mathrm{CH}$, Solorzano CC, Fidler IJ. Blockade of vascular endothelial growth factor receptor and epidermal growth factor receptor signaling for therapy of metastatic human pancreatic cancer. Cancer Res. 2002 Apr 1; 62(7):1996-2003.

24. Kuwahara K, Sasaki T, Kuwada Y, Murakami M, Yamasaki S, Chayama
K. Expressions of angiogenic factors in pancreatic ductal carcinoma: a correlative study with clinicopathologic parameters and patient survival. Pancreas. 2003 May:26(4):344-9.

25. Diamantidis M, Tsapournas G, Kountouras J, Zavos C. New aspects of regulatory signaling pathways and novel therapies in pancreatic cancer. Curr Mol Med. 2008 Feb;8(1):12-37.

26. Shultz DB, Pai J, Chiu W, Ng K, Hellendag MG, Heestand G, et al. A Novel Biomarker Panel Examining Response to Gemcitabine with or without Erlotinib for Pancreatic Cancer Therapy in NCIC Clinical Trials Group PA.3. PLoS One. 2016;11(1):e0147995

27. Chang YT, Chang MC, Wei SC, Tien YW, Hsu C, Liang PC, et al. Serum vascular endothelial growth factor/soluble vascular endothelial growth factor receptor 1 ratio is an independent prognostic marker in pancreatic cancer. Pancreas. 2008;37(2):145-50.

28. Truty MJ, Urrutia R. Basics of TGF-beta and pancreatic cancer. Pancreatology. 2007;7(5-6):423-35

29. Mohamed AA, Soliman H, Ismail M, Ziada D, Farid TM, Aref AM, et al. Evaluation of circulating ADH and MIC-1 as diagnostic markers in Egyptian patients with pancreatic cancer. Pancreatology. 2015 JanFeb;15(1): 34-9.

30. Wang X, Li Y, Tian H, Qi J, Li M, Fu C, et al. Macrophage inhibitory cytokine 1 (MIC-1/GDF15) as a novel diagnostic serum biomarker in pancreatic ductal adenocarcinoma. BMC Cancer. 2014 Aug 8;14:578.

31. Tonini G, Pantano F, Vincenzi B, Gabbrielli A, Coppola R, Santini D. Molecular prognostic factors in patients with pancreatic cancer. Expert Opin Ther Targets. 2007 Dec;11(12):1553-69.

32. Lv B, Zhang B, Hu XY, Zeng QD. Heparanase regulates VEGF-C expression and its clinical significance to pancreatic ductal cell adenocarcinoma. Oncol Lett. 2016 Feb;11(2):1327-34.

33. Kawase T, Yasui Y, Nishina S, Hara Y, Yanatori I, Tomiyama Y, et al. Fibroblast activation protein-alpha-expressing fibroblasts promote the progression of pancreatic ductal adenocarcinoma. BMC Gastroenterol. 2015 Sep 2:15:109.

34. Tanase CP. Caveolin-1: a marker for pancreatic cancer diagnosis. Expert Rev Mol Diagn. 2008 Jul;8(4):395-404.

35. Tanase CP, Dima S, Mihai M, Raducan E, Nicolescu MI, Albulescu L, et al. Caveolin-1 overexpression correlates with tumour progression markers in pancreatic ductal adenocarcinoma. J Mol Histol. 2009 Feb; 40(1):23-9.

36. Rahbari NN, Schmidt T, Falk CS, Hinz U, Herber M, Bork U, et al. Expression and prognostic value of circulating angiogenic cytokines in pancreatic cancer. BMC Cancer. 2011 Jul 5;11:286

37. Miyamoto M, Shimizu Y, Okada K, Kashii Y, Higuchi K, Watanabe A. Effect of interleukin-8 on production of tumor-associated substances and autocrine growth of human liver and pancreatic cancer cells. Cancer Immunol Immunother. 1998 Sep:47(1):47-57.

38. Sclabas GM, Fujioka S, Schmidt C, Li Z, Frederick WA, Yang W, et al. Overexpression of tropomysin-related kinase $\mathrm{B}$ in metastatic human pancreatic cancer cells. Clin Cancer Res. 2005 Jan 15;11(2 Pt 1): 440-9.

39. Li M, Zhang Y, Feurino LW, Wang H, Fisher WE, Brunicardi FC, et al. Interleukin-8 increases vascular endothelial growth factor and neuropilin expression and stimulates ERK activation in human pancreatic cancer. Cancer Sci. 2008 Apr;99(4):733-7.

40. Egberts JH, Cloosters V, Noack A, Schniewind B, Thon L, Klose S, et al. Anti-tumor necrosis factor therapy inhibits pancreatic tumor growth and metastasis. Cancer Res. 2008 Mar 1;68(5):1443-50.

41. Karayiannakis AJ, Syrigos KN, Polychronidis A, Pitiakoudis M, Bounovas A, Simopoulos K. Serum levels of tumor necrosis factoralpha and nutritional status in pancreatic cancer patients. Anticancer Res. 2001 Mar-Apr:21(2B):1355-8.

42. Macias I, Garcia-Perez S, Ruiz-Tudela M, Medina F, Chozas N, GironGonzalez JA. Modification of pro- and antiinflammatory cytokines and vascular-related molecules by tumor necrosis factor-a blockade in patients with rheumatoid arthritis. J Rheumatol. 2005;32(11):2102-8.

43. Apte RN, Dotan S, Elkabets M, White MR, Reich E, Carmi Y, et al. The involvement of IL-1 in tumorigenesis, tumor invasiveness, metastasis and tumor-host interactions. Cancer Metastasis Rev. 2006 Sep;25(3): 387-408.

44. Hanrahan EO, Lin HY, Kim ES, Yan S, Du DZ, McKee KS, et al. Distinct patterns of cytokine and angiogenic factor modulation and markers of benefit for vandetanib and/or chemotherapy in patients with non-small-cell lung cancer. J Clin Oncol. 2010 Jan 10;28(2):193-201.

45. Ebrahimi B, Tucker SL, Li D, Abbruzzese JL, Kurzrock R. Cytokines in pancreatic carcinoma: correlation with phenotypic characteristics and prognosis. Cancer. 2004 Dec 15;101(12):2727-36.

46. Carmeliet P, Jain RK. Molecular mechanisms and clinical applications of angiogenesis. Nature. 2011 May 19;473(7347):298-307. 